\title{
$\mathrm{M}|\mathrm{R}| \mathrm{S}$ Internet Journal Nitride Semiconductor Research
}

\section{Selective Etching of GaN from AlGaN/GaN and AIN/GaN Structures}

\author{
JA. Grenko ${ }^{1}$, CL. Reynolds $\mathrm{Jr}^{1}$, R. Schlesser ${ }^{1}, \mathrm{~K}$. Bachmann ${ }^{1}$, Z. Rietmeier ${ }^{1}$, Robert F. Davis ${ }^{1}$ and \\ Z. Sitar ${ }^{1}$ \\ ${ }^{1}$ Department of Materials Science and Engineering, North Carolina State University,
}

(Received Wednesday, April 7, 2004; accepted Wednesday, July 14, 2004)

Thick GaN layers as well as AlGaN/GaN and AlN/GaN heterostructures grown by metalorganic vapor phase epitaxy have been photoelectrochemically (PEC) etched in various dilute electrolytes, and bandgap-selective etching has been demonstrated in heterostructures. This result is a significant step forward in the fabrication of group III-nitride devices and one-dimensional photonic bandgap (PBG) structures in the deep UV. Based on initial results from thick GaN layers, a method was developed to achieve self-stopping selective etching of thin GaN layers in AlGaN/GaN and AlN/ GaN heterostructures. Selective PEC etching requires the use of a suitable light source with photon energies larger than the bandgap of GaN, but smaller than that of AlGaN or AlN, thus enabling selective hole generation in the GaN layers to be etched. Additionally, it is imperative to use an electrolyte that supports PEC etching of GaN without chemically etching AlGaN or AlN.

\section{Introduction}

Motivation for the work reported here was to develop a suitable processing technique for nanoscale patterning of multiple-period heterostructures, thus enabling the fabrication of AlN-based one-dimensional photonic bandgap structures (1D-PBGs). Potential applications include nanophotonic devices, UV photonic integrated circuits, and in particular, 1D-PBG-based non-linear optical frequency converters. The same processing technique is suitable for the fabrication of integrated, passive optical elements, including Bragg reflectors, interference filter structures, and for electronic devices.

For these applications, the ability to fabricate well defined, flat and plane-parallel interfaces with ultimate tolerances better than $0.1 \%$ is essential. The lack of chemical reactivity of group III nitrides to wet chemical etching has been well documented. [1] Porbaix [2] diagrams of AlN and GaN overlap significantly and therefore, selective etching by means of wet chemical methods is not sufficiently selective. Alternatively, Wang et al. [3] have demonstrated selective etching of $\mathrm{GaN}$ from InGaN to create high reflectivity semiconductor/air distributed Bragg reflectors using chlorine-based reactive ion etching (RIE). However, their etching process did not consider high aspect ratios and resulted in distinct inclination of the sidewalls of their Bragg reflectors.
In PEC etching, electron-hole pairs are photogenerated in a semiconductor placed in an electrolytic solution and illuminated with photons of energy greater than its bandgap. Stonas et al. [4] demonstrated bandgapselectivity by etching a thin layer of lower bandgap InGaN sandwiched between thick n-GaN layers. PEC etching has become a valuable tool for the fabrication of high power devices such as $\mathrm{AlGaN} / \mathrm{GaN}$ high electron mobility transistors (HEMTs) and heterojunction bipolar transistors (HBTs) because it enables selective lateral etching of material by virtue of its bandgap selectivity. Improved I-V characteristics in current aperture vertical electron transistors (CAVET) have been demonstrated [5] by using a PEC process to remove the insulating layer of conventional CAVET structures [6] thereby eliminating a path for leakage current. Khare and $\mathrm{Hu}$ [7] examined the etch selectivity of $\mathrm{n}^{+}$GaAs on $\mathrm{p}$-GaAs and NID GaAs substrates and found significant etch rate differences in $\mathrm{n}^{+}$and $\mathrm{p}^{+}$GaAs homostructures. Flynn [8] extended the utility of this dopant-selectivity in the fabrication of $1.3 \mathrm{~mm}$ LEDs when he demonstrated improved coupling efficiency to optical fibers by PECetching n-InGaAsP into curved lenses. Thus, PEC etching has been demonstrated to be an important fabrication technique for electronic and photonic devices. 
Since PEC etching requires the generation of holes to form oxides with the semiconductor and subsequent dissolution of those oxides in the electrolyte, selection of an appropriate electrolyte is a primary concern. Minsky et al. [9] demonstrated that PEC etching of nonintentionally doped (NID) GaN could be achieved in solutions of $\mathrm{HCl}: \mathrm{H}_{2} \mathrm{O}(1: 10)$ and solutions of $45 \%$ $\mathrm{KOH}: \mathrm{H}_{2} \mathrm{O}$ (1:3). Additionally, Peng showed the solubility of $\mathrm{GaN}$ in electrolytes of $\mathrm{H}_{3} \mathrm{PO}_{4}$ and $\mathrm{KOH}$ in the $\mathrm{pH}$ regime from -1 to 2 and 11 to 15 , respectively. [10] A further refinement of PEC-etching using dilute electrolyte solutions of $0.04 \mathrm{M} \mathrm{KOH}$ to generate smooth $\mathrm{GaN}$ surfaces when etched in the diffusion-controlled regime has been reported. [11] Selective PEC etching of heterostructures requires the use of light with energy higher than the bandgap of the material to be etched, yet lower than the bandgap of material to be preserved. The photons are absorbed in the material to be etched and electron-hole pairs are generated. The holes are transported to the semiconductor-electrolyte interface for bond-breaking reactions with the semiconductor. Meanwhile, the electrons are swept to the cathode to reduce the probability of unwanted electron-hole recombination. In addition to adequate illumination, a good electrical contact to the semiconductor (anode), as well as a sufficiently large cathode for effective carrier sweep-out are required.

\section{Experimental}

Initial etching experiments were performed on $\mathrm{GaN}$ samples grown by metalorganic vapor phase epitaxy onto sapphire substrates. GaN layers were typically 5 $\mu \mathrm{m}$ thick, and either not intentionally doped (NID) with a carrier concentration of $\sim 5 \times 10^{17} \mathrm{~cm}^{-3}$, or, alternatively highly-doped with a carrier concentration of $\sim 1 \mathrm{x}$ $10^{19} \mathrm{~cm}^{-3}$. $300 \mathrm{~nm}$ thick Ti contacts were deposited either as square pads with $10 \times 10 \mu \mathrm{m}^{2}$ to $50 \times 50 \mu \mathrm{m}^{2}$ openings or $10 \mu \mathrm{m}$ parallel lines with $10 \mu \mathrm{m}$ openings. The material was then cleaved into approximately $5 \mathrm{~mm}$ $\mathrm{x} 5 \mathrm{~mm}$ samples.

In order to investigate wet etching properties without illumination, films of $\mathrm{AlN}$ and $\mathrm{GaN}$ were placed in separate beakers of $1: 10 \mathrm{HCl}: \mathrm{H}_{2} \mathrm{O}, 1 \mathrm{M} \mathrm{KOH}$ and $1: 25$ $\mathrm{H}_{3} \mathrm{PO}_{4}: \mathrm{H}_{2} \mathrm{O}$ covered with aluminum foil for a $24 \mathrm{hr}$ time period at room temperature. Bright-field optical microscopy revealed no evidence of etch pits. Although we used these molarities and concentrations of the electrolytes as upper limits, we did investigate and will report on other electrolytes investigated in the course of this study.

An Oriel high-pressure mercury arc lamp, Model 66021 (1000 Watt), with spectral emission lines at 297,
313 and $365 \mathrm{~nm}$ provided photon energies near and above the bandgap of GaN. A one meter long optical light guide fiber was used to couple the light to the sample and a lens reduced the spot size to $1.3 \mathrm{~cm}^{2}$. A cold mirror was also used to eliminate infrared illumination thereby reducing unwanted heating of samples. After spectral and transmission loss corrections, the estimated illumination intensity at $365 \mathrm{~nm}$ was $30 \mathrm{~mW} \cdot \mathrm{cm}^{-2}$. The experimental apparatus was similar to that described in the literature. [9] Since the electrolytes used were highly acidic or basic, samples were mounted on a Teflon block. A stainless steel washer was used to increase the contact area to the sample (anode). A gold wire was wound around the screw securing the GaN sample to the Teflon block, and a 6" long $1 \mathrm{~mm} \mathrm{Pt}$ wire wound to a coil of $8 \mathrm{~mm}$ diameter served as the cathode.

\section{Results}

The photocurrent, which is proportional to the etch rate at the semiconductor-electrolyte interface, was measured with a digital multimeter and varied from 30 to $600 \mu \mathrm{A}$ for the dilute electrolytes $\mathrm{H}_{3} \mathrm{PO}_{4}, \mathrm{KOH}$, and $\mathrm{HCl}$, while the dark current for all electrolytes was $<2$ $\mu \mathrm{A}$. The photocurrent is a measure of the oxidation reactions being induced by holes $\left(\mathrm{h}^{+}\right)$in the valence band. The presence of holes at the semiconductor-electrolyte interface allows ionic dissolution of the semiconductor. Youtsey [11] proposed that the following oxidation reaction is responsible for the decomposition of $\mathrm{GaN}$,

$$
2 G a N+6 h^{+} \rightarrow 2 G a^{3+}+N_{2} .
$$

Bubble formation was observed in these experiments. The formation of bubbles on the sample surface during etching had been previously suggested [11] as evidence for the release of $\mathrm{N}_{2}$.

The resultant photocurrent data for our initial choice of chemistries and geometries are shown in Figure 1. Note the stability of the photocurrent with etch time. It is evident from inspection of the data that the magnitude of the photocurrent is specific to the choice of the electrolyte. But more importantly, the data suggest that the magnitude of the photocurrent in solutions of the same electrolyte is a function of the ratio between the masked and unmasked illuminated area for the geometries used in these studies. Integration of the photocurrent vs. time curve estimates that the total charge through the cell was $0.2-0.5 \mathrm{C}$, depending on the etch time and chemistry.

After PEC etching, samples were examined with a field emission scanning electron microscope (FESEM) to verify that GaN etching did indeed occur, and if so, to determine the resultant etch depth. For the specific example of a $1 \mathrm{hr}$ etch using $1: 25 \mathrm{H}_{3} \mathrm{PO}_{4}: \mathrm{H}_{2} \mathrm{O}$ we observed an average photocurrent of $40 \mu \mathrm{A}$. The result- 
ant etch depth was $1.25 \mu \mathrm{m}$ for an effective etch rate of $21 \mathrm{~nm} \cdot \mathrm{min}^{-1}$. The observed average photocurrent, $\mathrm{I}_{\mathrm{ph}}$, for each of the electrolytes gave a reasonable prediction of the etch rates based on Faraday's Law, from which the etch rate can be expressed as

$$
\frac{h}{t}=\frac{\alpha \cdot J \cdot M_{w}}{n \cdot F \cdot \rho}
$$

where $h$ is the etch depth, $t$ the etch time, $\alpha$ the collection efficiency at the cathode, $\mathrm{J}$ the current density, $\mathbf{M}_{\mathrm{W}}$ the molecular weight, F Faraday's constant, and $\rho$ the density of GaN. However, since we have no accurate determination for the illuminated area and the current density can be written as $\mathrm{J}=\mathrm{I}_{\mathrm{ph}} / \mathrm{A}$, where A is the illuminated area, Equation (1) can be written as,

$$
\frac{h}{t}=\frac{\alpha^{\prime} \cdot I_{p h} \cdot M_{w}}{n \cdot F \cdot \rho}
$$

where $\alpha^{\prime}$ is defined as $\alpha /$ A. Using the etch rate for 1:25 $\mathrm{H}_{3} \mathrm{PO}_{4}: \mathrm{H}_{2} \mathrm{O}$ to determine $\alpha^{\prime}$ in Equation (2), we can then use the measured photocurrents of 60 and $150 \mu \mathrm{A}$ to predict the PEC-etch rates for 2:25 $\mathrm{H}_{3} \mathrm{PO}_{4}: \mathrm{H}_{2} \mathrm{O}$ and $0.04 \mathrm{M} \mathrm{KOH}$ electrolytes without any adjustable parameters. The calculated etch rates of 31 and $72 \mathrm{~nm} \cdot \mathrm{min}^{-1}$ are in reasonable agreement with the etch depths per unit time (33 and $51 \mathrm{~nm} \cdot \mathrm{min}^{-1}$ ) as measured by FESEM and recorded in Table 1. The difference in the $\mathrm{KOH}$ results suggest that there may be slightly different cathode efficiencies based on the selection of the electrolyte.

Table 1 summarizes all the electrolytes that were investigated. Although the measured photocurrent for 1:10 HCl: $\mathrm{H}_{2} \mathrm{O}$ was high, there was no evidence of etching. In general and with the exception of the 1:10 $\mathrm{HCl}: \mathrm{H}_{2} \mathrm{O}$ sample, FESEM inspection of the samples etched in electrolytes showing no evidence of etching revealed extensive patches of oxide across the semiconductor surface. These data suggested that excessive oxide formation most likely inhibited PEC etching of the $\mathrm{GaN}$ for these electrolytes.

Figure 2 shows a plot of photocurrent vs. illumination intensity for $\mathrm{GaN}$ etched in $\mathrm{KOH}$. In the region of low illumination intensities, the photocurrent behavior is linear which is indicative of a carrier-limited regime, which is below $650 \mathrm{~W}$ for our experiments. Here, the reaction rate is limited only by electron-hole pair generation. As the illumination is further increased, the mea- sured photocurrent becomes sublinear as it approaches the diffusion-limited etch regime. Due to the intensity limitations of our experimental apparatus, saturation, which is indicative of a diffusion-limited process, was never reached. However, it must be noted that the ability to generate holes at the semiconductor-electrolyte interface appears to be correlated to the n-carrier concentration of the material as is indicated by the 2.5 -fold increase in etch rate for the material with a donor concentration of $\sim 1 \times 10^{19} \mathrm{~cm}^{-3}$, as seen in Table I. In contrast to NID GaN and for nearly identical photocurrents, the PEC etching of this material resulted in whisker-free surfaces. These observations are consistent with the decrease in Debye length of the semiconductor as the doping density increases. A higher donor concentration confines the holes closer to the semiconductor surface thereby enabling more efficient etching.

While both $\mathrm{KOH}$ and $\mathrm{H}_{3} \mathrm{PO}_{4}$ chemistries resulted in well-defined patterns with vertical sidewalls in the thick film GaN material, GaN whiskers typically remained within the etched area. This observation is consistent with earlier reports that free-standing GaN whiskers remain on the surface for carrier-limited etch conditions. [12] Whiskers have been observed previously for a narrow range of $\mathrm{KOH}$ concentrations near $0.02 \mathrm{M}$ [11] and for $\mathrm{H}_{3} \mathrm{PO}_{4}$ at $\mathrm{pH}=3$. [10] It has also been reported that whisker-free surfaces can be achieved for PEC etching under diffusion-controlled etch conditions in $\mathrm{KOH}$. [13] The ability to deliver the electrolyte to the surface for dissolution of the GaN can be assisted by stirring the solution. However, in all cases stirring the solution caused an increase in the surface roughness due to highly localized etch rates at the semiconductor surface unless the concentration of the electrolyte was significantly reduced. Since the fabrication of PBG structures requires smooth interfaces, the electrolyte solutions were not stirred in this work. With the exception of the high carrier concentration $\left(\sim 1 \times 10^{19} \mathrm{~cm}^{-3}\right) \mathrm{GaN}$, whisker-free GaN surfaces could not be achieved by simply PEC etching in an appropriate electrolyte. In order to remove the whiskers after completion of the PEC etch, a wet chemical etch was performed for 15 to $30 \mathrm{~min}$ in $2 \mathrm{M} \mathrm{KOH}$. Although some whiskers remained after 15 min (Figure 3a), a 30 min clean-up etch resulted in a whisker-free surface with no deleterious effect on the vertical sidewalls (Figure $3 b$ ). Note the lack of inclination the sidewall, which is key feature for the fabrication of nanophotonic devices. Another alternative is to use highly doped, $\sim 10^{19} \mathrm{~cm}^{3}{ }^{3}, \mathrm{GaN}$, in which no whiskers were observed for our etch conditions.

The heterostructure materials used in this study were grown by MOVPE on sapphire and $6 \mathrm{H} \mathrm{SiC} \mathrm{(0001)} \mathrm{sub-}$ strates. These structures consisted of alternating stacks 
of $\mathrm{AlN}$ and $\mathrm{GaN}$ or $\mathrm{AlGaN}$ and GaN, comprising 5 layers, each of 100-200 nm thickness. In order to establish an electrical contact to the GaN layers, a shallow-angle, $5 \mathrm{~mm}$ wide wedge was polished off each sample, and a $300 \mathrm{~nm}$ thick Ti contact was deposited. This approach was more effective in the AlGaN/GaN heterostructures than the AlN/GaN heterostructures since the insulating layers of AlN diminish the effective cross-sectional contact to the GaN material.

$\mathrm{AlN} / \mathrm{GaN}$ and $\mathrm{AlGaN} / \mathrm{GaN}$ heterostructures prepared in this manner required no further photolithographic patterning. The metallized edge of the sample was fastened to the Teflon block using a stainless steel washer. We used electrolytes of $\mathrm{KOH}$ and $\mathrm{H}_{3} \mathrm{PO}_{4}$ and illuminated the samples with $750 \mathrm{~W}$ of illumination. For the AlN/GaN heterostructures etched in $\mathrm{KOH}$, the appearance of cones below each of the GaN layers was evidence that the AlN layers were being selectively etched with respect to the GaN layers. Switching electrolytes from $\mathrm{KOH}: \mathrm{H}_{2} \mathrm{O}$ to $\mathrm{H}_{3} \mathrm{PO}_{4}: \mathrm{H}_{2} \mathrm{O}$ circumvented the solubility issues. PEC etching the AlGaN/GaN heterostructures resulted in the desired effect of etching the $200 \mathrm{~nm}$ GaN layers preferentially while leaving the AlGaN layers intact, as shown in Figure 4. There is evidence of whisker formation in the GaN layers; in order to provide whisker-free surfaces, longer PEC-etching or the use of more highly doped $\mathrm{GaN}$ yielding accelerated etch rates would be required.

Although it is clear by examination of the Pourbaix [2] diagrams of AlN and GaN that etching an AlN/GaN heterostructure in $\mathrm{KOH}$ should result in solubility of the AlN in preference to $\mathrm{GaN}$, low photocurrent was observed for the AlN/GaN samples. To address this issue, a simple AlN/GaN heterostructure having $200 \mathrm{~nm}$ $\mathrm{GaN}$ sandwiched between $100 \mathrm{~nm}$ AlN grown on a sapphire substrate was designed and an alternative means of establishing contact to the GaN layers was employed. The first step was a full surface metallization of $300 \mathrm{~nm}$ Ti onto the AlN/GaN substrate followed by a photolithographically generated metal mask in order to selectively wet-etch channels into the top AlN layer. Afterward, the sample was etched in dilute buffered-HF to remove the Ti mask. The full surface metallization with $300 \mathrm{~nm}$ Ti was then repeated. This processing generated areas where the GaN layers were in direct contact with the $\mathrm{Ti}$ mask. By employing backside illumination through the sapphire substrate, $\mathrm{GaN}$ in the heterostructure was selectively etched in 2:25 $\mathrm{H}_{3} \mathrm{PO}_{4}: \mathrm{H}_{2} \mathrm{O}$. Figure 5 shows that this fabrication sequence and the selection of the appropriate electrolyte result in the desired selective etching of $200 \mathrm{~nm} \mathrm{GaN}$ layer from the $100 \mathrm{~nm}$ AlN layers. Additional effort is required to ensure smooth interfaces.

\section{Conclusions}

To our knowledge, this is the first demonstration of selective etching of $\mathrm{GaN}$ from $\mathrm{AlN}$ and $\mathrm{AlGaN}$ layers. Significant differences in etch rate between the high and low carrier concentration material investigated in this study suggest that it will be beneficial to increase the $n$ carrier concentration in the GaN material to further improve selectivity. Additionally, it should be investigated whether thin p-type layers are indeed resistant to PEC etching. This work could be confirmed using thin $\mathrm{GaN}$ homostructures of alternating $\mathrm{p}$ - and $\mathrm{n}$ - type material. And finally, p-doping the thin AlN and AlGaN layers within the AlN/GaN and $\mathrm{AlGaN} / \mathrm{GaN}$ heterostructures while keeping the n-carrier concentration of the GaN high may provide even greater selectivity. To our knowledge, successful p-doping of these layers has not be achieved to date.

\section{REFERENCES}

[1] C. B. Vartuli, S. J. Pearton, C. R. Abernathy, J. D. MacKenzie, Sol. St. Electr. 41, 1947 (1997).

[2] Pourbaix, M. Atlas of Electrochemical Equilibria in Aqueous Solutions, 2nd edition, 1974. pp 171 and 431

[3] H. Wang, M. Kumagai, T. Tawara, T. Nishida, T. Akasaka, N. Kobayashi, T. Saitoh, Appl. Phys. Lett. 81, 4703 (2002).

[4] A. R. Stonas, T. Marglith, S. P. Denbaars, L. A. Coldren, E. L. Hu, Appl. Phys. Lett. 78, 1945 (2001).

[5] Y. Gao, Ar. R. Stonas, I. Ben-Yaacov, U. Mishra, S. P. DenBaars, E. L. Hu, Electron. Lett. 39, 1 (2003).

[6] I. Ben-Yaacov, Y.K. Seck, S.P. DenBaars, E.L. Hu, and U.K. Mishra, Mat. Res. Soc. Symp. Proc. 764,C4.8.1(2003)

[7] R. Khare, E. Hu, J. Electrochem. Soc. 138, 1516 (1991).

[8] E. J. Flynn, unpublished

[9] M. S. Minsky, M. White, E. L. Hu , Appl. Phys. Lett. 68, 1531-1533 (1996).

[10] L. -H. Peng, C. -W. Peng, J. -K. Ho, C. -N. Huang, C. -Y. Chen, Appl. Phys. Lett. 72, 939 (1998).

[11] C. Youtsey, I. Adesida, G. Bulman, Appl. Phys. Lett. 71, 2151 (1997).

[12] C. Youtsey, L. T. Romano, I. Adesida, Appl. Phys. Lett. 73, 797 (1998).

[13] C. Youtsey, I. Adesida, L. T. Romano, G. Bulman, Appl. Phys. Lett. 72, 560 (1998). 


\section{FIGURES}
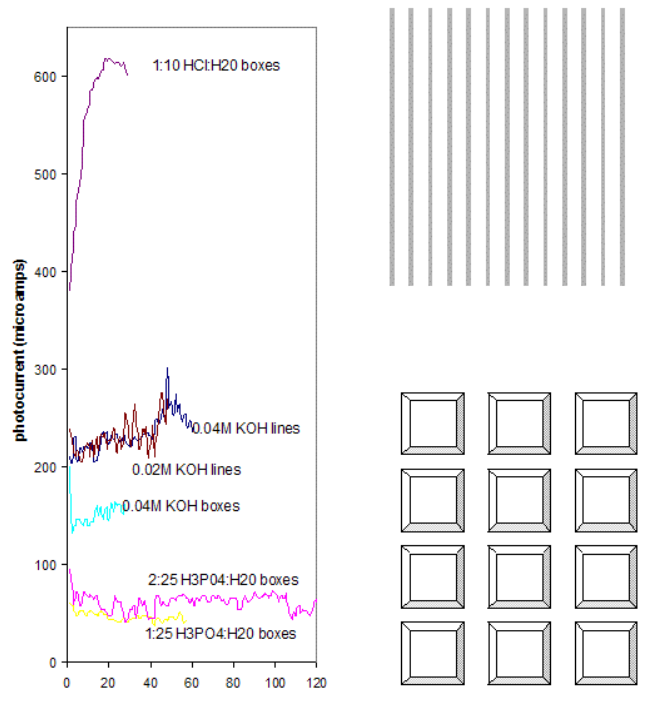

Figure 1. Photocurrent vs. etch time for electrolytes of $\mathrm{KOH}$, $\mathrm{H}_{3} \mathrm{PO}_{4}$, and $\mathrm{HCl}$ (left) and two types of mask geometry used in this study.

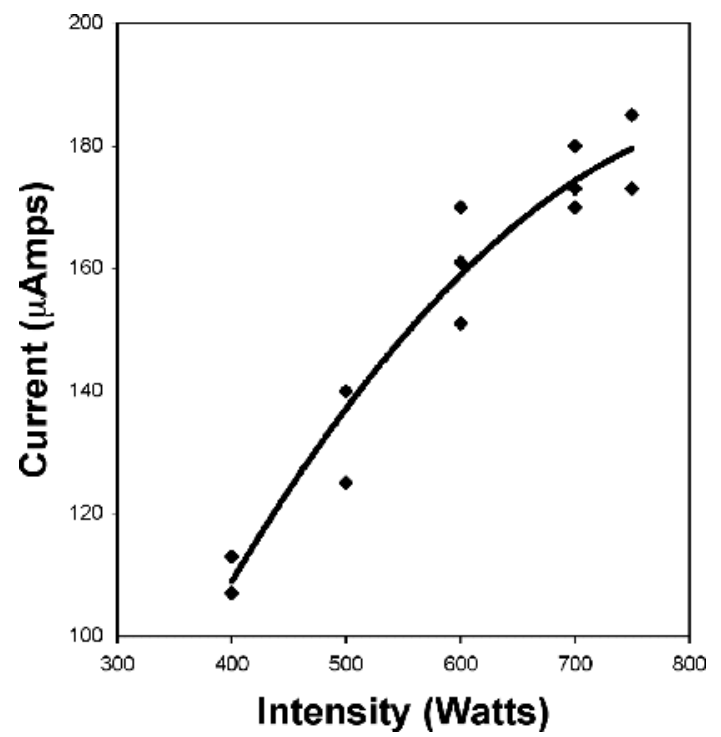

Figure 2. Photocurrent behavior in the carrier-limited regime.

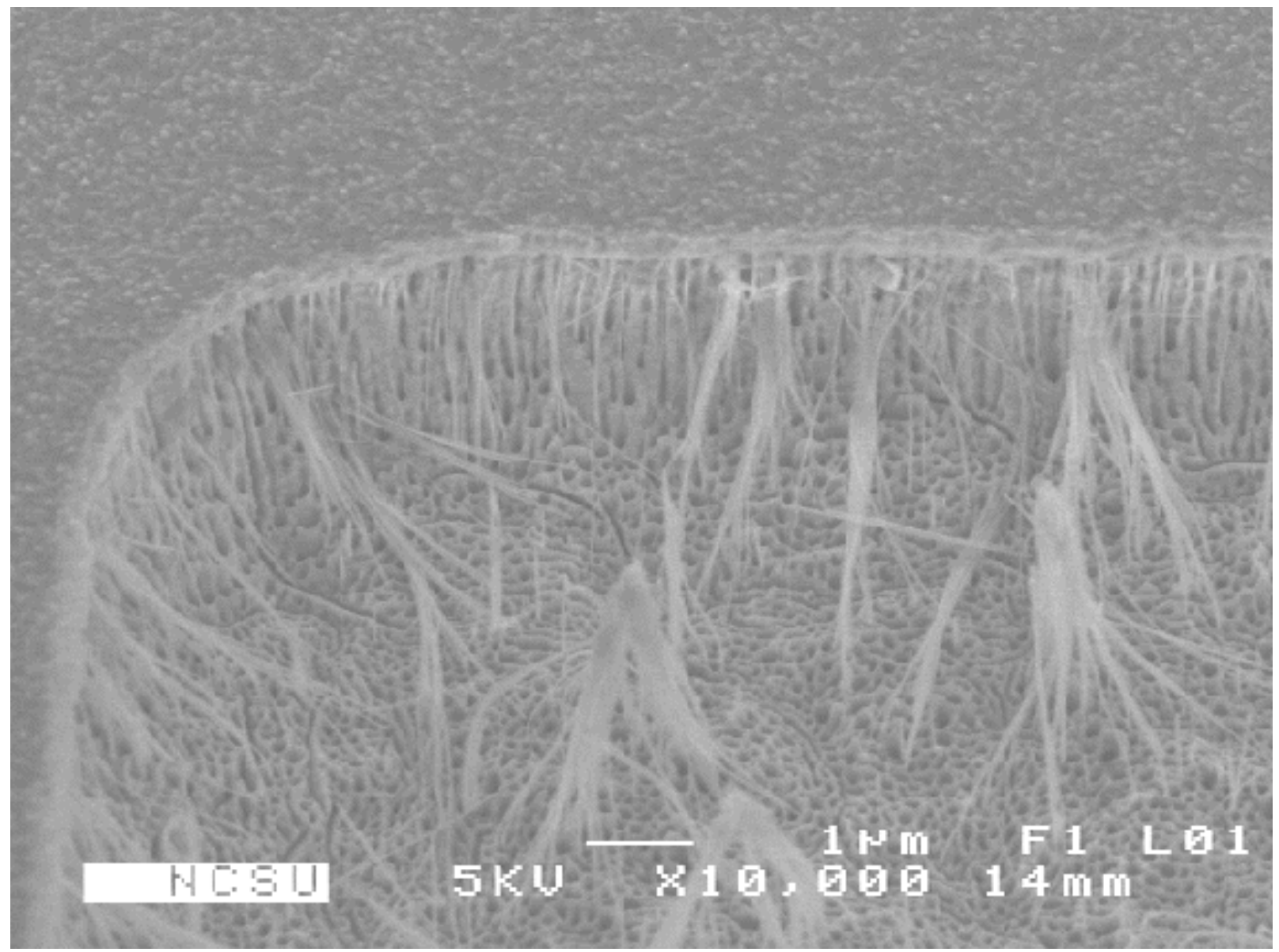

Figure 3a. Whiskers remaining after 15 min. clean-up etch. 


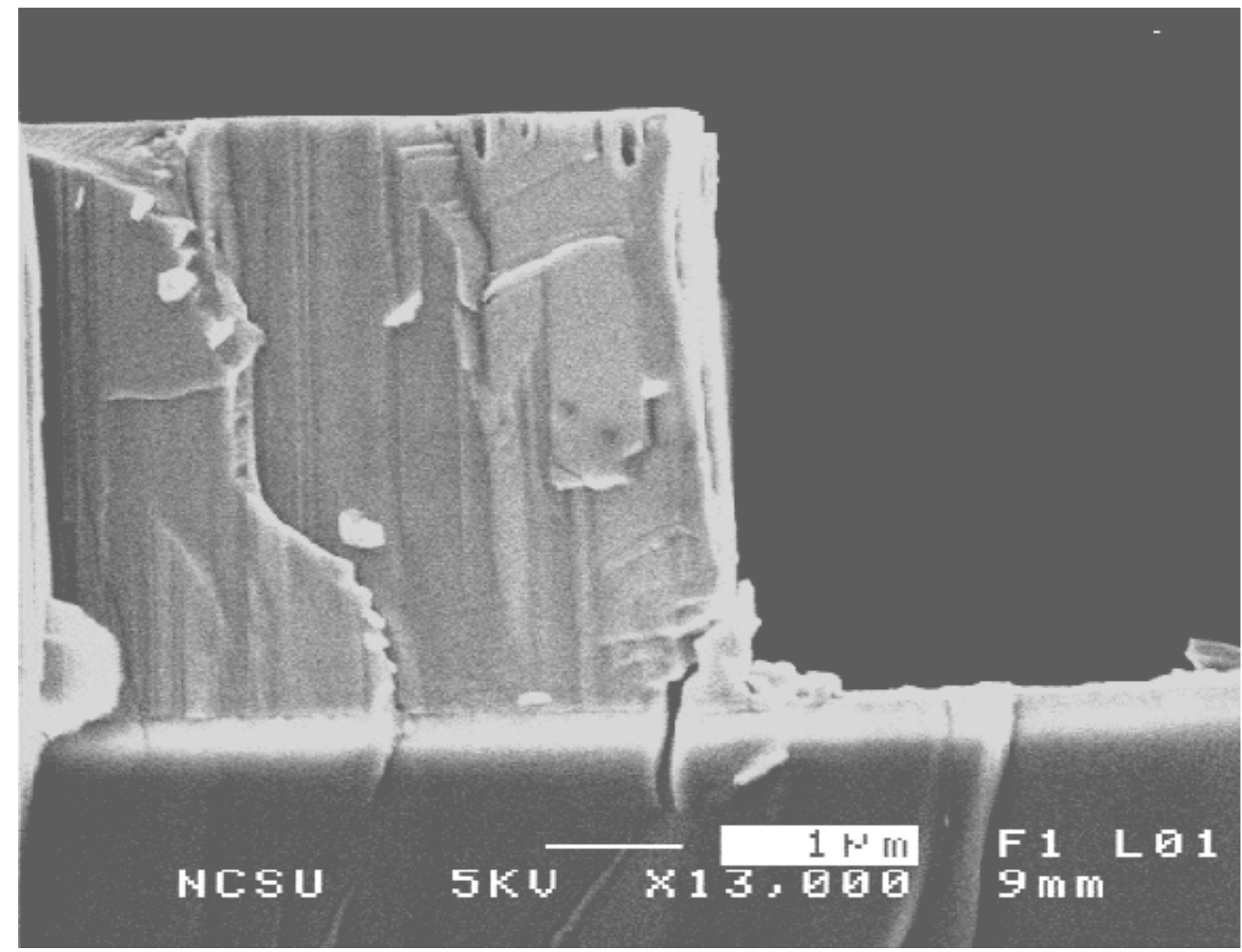

Figure 3b. Line-patterned $\mathrm{GaN}$ after $3 \mathrm{hr}$. PEC etch in $\mathrm{H}_{3} \mathrm{PO}_{4}: \mathrm{H}_{2} \mathrm{O}$ at $\mathrm{pH}=3$ followed by 30 min. clean-up etch in $2 \mathrm{M} \mathrm{KOH}$. 


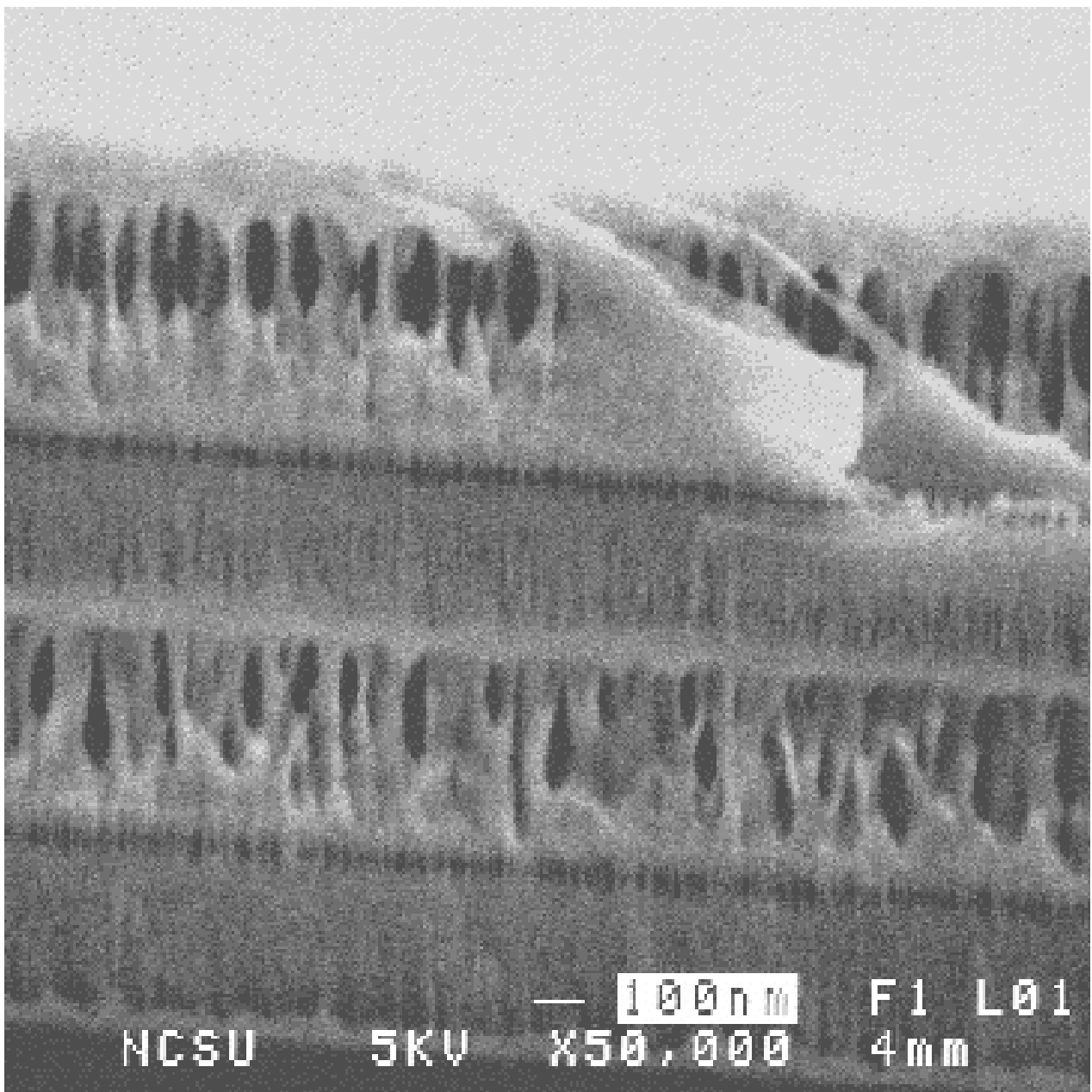

Figure 4. PEC-etched AlGaN/GaN heterostructure showing good selectivity between the AlGaN and the GaN layers. 

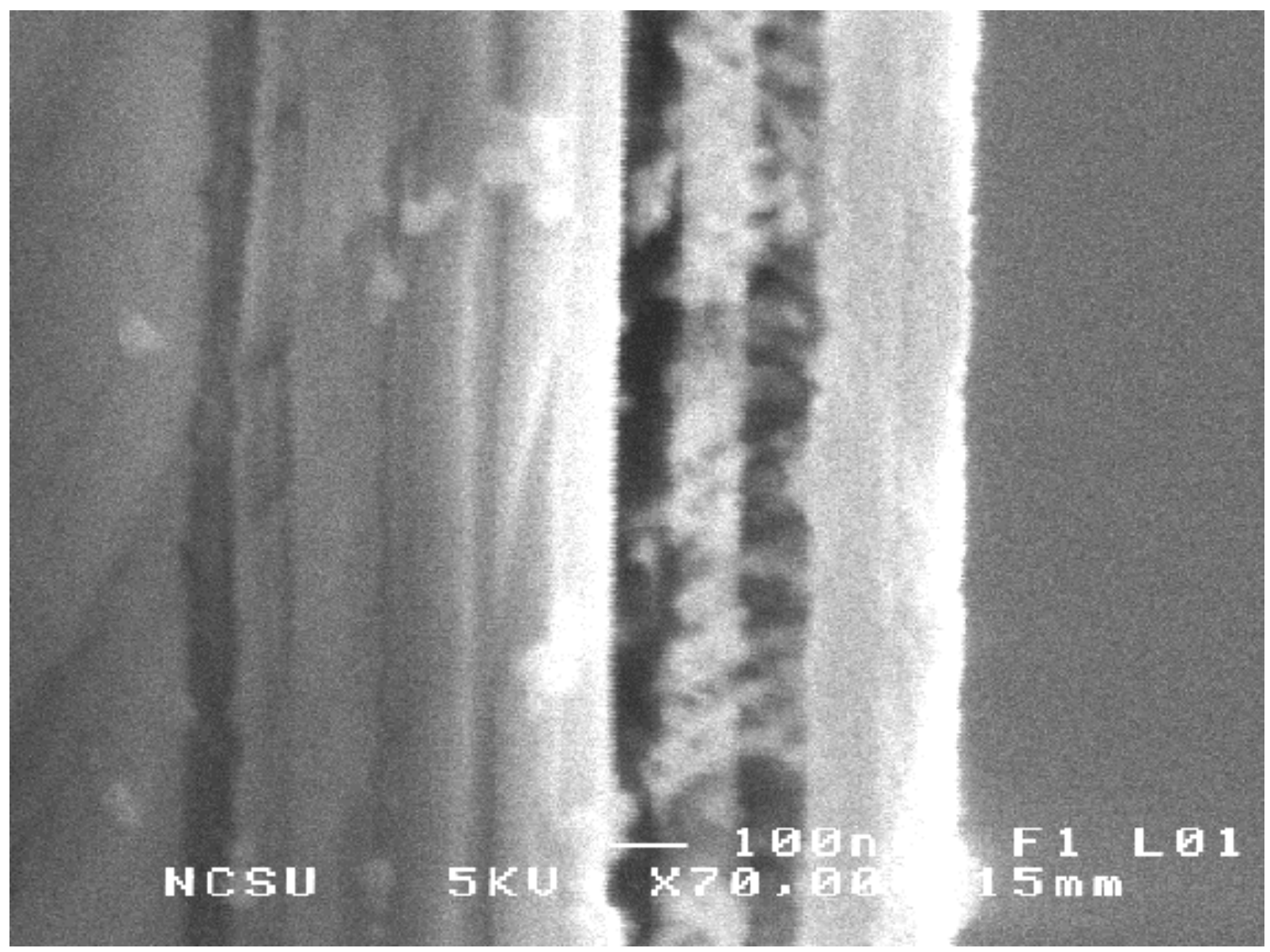

Figure 5. PEC-etched AlN/GaN/AIN sample after 3 hr. etch in 2:25 $\mathrm{H}_{3} \mathrm{PO}_{4}: \mathrm{H}_{2} \mathrm{O}$.

\section{TABLES}

Table 1. Comparison of room temperature PEC etch rates for $\mathrm{GaN}$ and $\mathrm{AlN}$ under $\mathrm{Hg}$ arc illumination.

\begin{tabular}{|l|l|l|c|c|}
\hline Electrolyte & Concen. & $\mathrm{I}_{\mathrm{ph}}[\mu \mathrm{A}]$ & GaN etch rate [nm/min] & AlN etch rate [nm/min] \\
\hline $\mathrm{KOH}$ & $0.02 \mathrm{M}$ & 150 & 42 & 0 \\
\hline $\mathrm{KOH}$ & $0.04 \mathrm{M}$ & 150 & 51 & 0 \\
\hline $\mathrm{H}_{3} \mathrm{PO}_{4}: \mathrm{H}_{2} \mathrm{O}$ & $1: 25$ & 40 & 21 & 0 \\
\hline $\mathrm{H}_{3} \mathrm{PO}_{4}: \mathrm{H}_{2} \mathrm{O}(\mathrm{NID} \mathrm{GaN})$ & $2: 25$ & 60 & 33 & 0 \\
\hline $\mathrm{H}_{3} \mathrm{PO}_{4}: \mathrm{H}_{2} \mathrm{O}\left(\sim 1 \mathrm{E} 19 \mathrm{~cm}^{-2} \mathrm{GaN}\right)$ & $2: 25$ & 60 & 83 & 0 \\
\hline $\mathrm{HCl}: \mathrm{H}_{2} \mathrm{O}$ & $1: 10$ & 600 & 0 & 0 \\
\hline $\mathrm{H}_{2} \mathrm{SO}_{4}: \mathrm{H}_{2} \mathrm{O}$ & $1: 25$ & 20 & 0 & - \\
\hline $\mathrm{B}_{4} \mathrm{~K}_{2} \mathrm{O}_{7}$ & $0.1 \mathrm{M}$ & 175 & 0 & - \\
\hline $\mathrm{B}_{4} \mathrm{Na}_{2} \mathrm{O}_{7}$ & $0.1 \mathrm{M}$ & 175 & 0 & - \\
\hline $\left.\mathrm{C}_{4} \mathrm{H}_{6} \mathrm{O}_{6}: \mathrm{NaOH}\right): \mathrm{H}_{2} \mathrm{O}$ & $2: 100$ & 30 & 0 & - \\
\hline $\left.\mathrm{HBrpH}_{2} .67\right): \mathrm{H}_{2} \mathrm{O}$ & $2: 100$ & 45 & 0 & 0 \\
\hline
\end{tabular}

